\title{
BOUNDARY TWO-PARAMETER EIGHT-STATE SUPERSYMMETRIC FERMION MODEL AND BETHE ANSATZ SOLUTION
}

\author{
Anthony J. Bracken, Xiang-Yu Ge, Yao-Zhong Zhang \\ AND HUAN-QIANG ZHOU
}

\begin{abstract}
The recently introduced two-parameter eight-state $U_{q}[g l(3 \mid 1)]$ supersymmetric fermion model is extended to include boundary terms. Nine classes of boundary conditions are constructed, all of which are shown to be integrable via the graded boundary quantum inverse scattering method. The boundary systems are solved by using the coordinate Bethe ansatz and the Bethe ansatz equations are given for all nine cases.
\end{abstract}

\section{INTRODUCTION}

Low dimensional integrable quantum systems, with or without open boundary conditions, which describe strongly correlated fermions [8] form an important class of lattice integrable models, which have attracted much international attention (see, for example, $[2,10,18,19,6,3,11,1,14]$ and references therein). Recently, trying to extend the existing two component electron models to multi-component cases, we proposed $[12,9]$ an eight-state integrable model and its two-parameter (or $q$-deformed) version with Lie superalgebra $g l(3 \mid 1)$ and quantum superalgebra $U_{q}[g l(3 \mid 1)]$ symmetries, respectively. One of the features of these two models is that they contain correlated single-particle and pair hoppings, uncorrelated triple-particle hopping and two- and three-particle on-site interactions. By eight-state, we mean that at a given lattice site $j, j=1,2, \ldots L$, there are eight possible fermionic states:

$$
\begin{aligned}
& |0\rangle, \quad c_{j, 1}^{\dagger}|0\rangle, \quad c_{j, 2}^{\dagger}|0\rangle, \quad c_{j, 3}^{\dagger}|0\rangle \text {, } \\
& c_{j, 1}^{\dagger} c_{j, 2}^{\dagger}|0\rangle, \quad c_{j, 1}^{\dagger} c_{j, 3}^{\dagger}|0\rangle, \quad c_{j, 2}^{\dagger} c_{j, 3}^{\dagger}|0\rangle, \quad c_{j, 1}^{\dagger} c_{j, 2}^{\dagger} c_{j, 3}^{\dagger}|0\rangle,
\end{aligned}
$$

where $c_{j, \alpha}^{\dagger}\left(c_{j, \alpha}\right)$ denotes a fermionic creation (annihilation) operator which creates (annihilates) a fermion of species $\alpha=1,2,3$ at site $j$; these operators satisfy the anticommutation relations given by $\left\{c_{i, a}^{\dagger}, c_{j, \beta}\right\}=\delta_{i j} \delta_{\alpha \beta}$.

\section{Received 17th September, 1998}

X.-Y. Ge and H.-Q. Zhou are supported by OPRS and UQPRS. Y.-Z. Zhang is supported by a QEII Fellowship Grant from the Australian Research Council.

Copyright Clearance Centre, Inc. Serial-fee code: 0004-9729/99 \$A2.00+0.00. 
Recently we formulated in $[4,5]$ a general and fully supersymmetric version of the boundary inverse scattering method $[15,13,7]$, and constructed a large number of integrable boundary conditions $[16,17]$ for various models of strongly correlated fermions. In this paper, we continue our study of open boundary conditions and consider the integrable eight-state fermion model with $U_{q}[g l(3 \mid 1)]$ symmetry. We present nine classes of boundary conditions for this model, all of which are shown to be integrable by the graded boundary QISM $[4,5]$. We solve the boundary systems by using the coordinate Bethe ansatz method and derive the Bethe ansatz equations for all nine cases.

\section{Open Boundary Conditions}

We consider the following Hamiltonian with boundary terms

$$
H=\sum_{j=1}^{L-1} H_{j, j+1}(g, \kappa)+H_{l t}^{\text {boundary }}+H_{r t}^{\text {boundary }},
$$

where $H_{l t}^{\text {boundary }}, H_{r t}^{\text {boundary }}$ are respectively left and right boundary terms whose explicit forms are given below, and $H_{j, j+1}$ is the Hamiltonian density of the two-parameter eightstate supersymmetric fermion model [9]

$H_{j, j+1}(g, \kappa)=-\sum_{\alpha}\left(c_{j, \alpha}^{\dagger} c_{j+1, \alpha}+\right.$ h.c. $) \exp \left\{-\frac{1}{2}(\eta+\kappa) \sum_{\beta(\neq \alpha)} n_{j+\theta(\beta-\alpha), \beta}-\frac{1}{2}(\eta-\kappa)\right.$

$$
\begin{gathered}
\left.\sum_{\beta(\neq \alpha)} n_{j+1-\theta(\beta-\alpha), \beta}+\frac{\zeta}{2} \sum_{\beta \neq \gamma(\neq \alpha)}\left(n_{j, \beta} n_{j, \gamma}+n_{j+1, \beta} n_{j+1, \gamma}\right)\right\} \\
-\frac{\sinh \kappa}{2 \sinh \kappa(g+1)} \sum_{\alpha \neq \beta \neq \gamma}\left(c_{j, \alpha}^{\dagger} c_{j, \beta}^{\dagger} c_{j+1, \beta} c_{j+1, \alpha}+\text { h.c. }\right) \\
\exp \left\{-\left(\frac{\xi}{2}-\operatorname{sign}(\gamma-2) \kappa\right) n_{j, \gamma}-\left(\frac{\xi}{2}+\operatorname{sign}(\gamma-2) \kappa\right) n_{j+1, \gamma}\right\} \\
-\frac{2 \cosh \kappa \sinh ^{2} \kappa}{\sinh \kappa(g+1) \sinh \kappa(g+2)}\left(c_{j, 1}^{\dagger} c_{j, 2}^{\dagger} c_{j, 3}^{\dagger} c_{j+1,3} c_{j+1,2} c_{j+1,1}+\text { h.c. }\right) \\
+e^{\kappa g} n_{j}+e^{-\kappa g} n_{j+1}-\frac{\sinh \kappa}{2 \sinh \kappa(g+1)} \sum_{\alpha \neq \beta}\left(n_{j, \alpha} n_{j, \beta}+n_{j+1, \alpha} n_{j+1, \beta}\right) \\
+\frac{2 \cosh \kappa(g+1) \sinh ^{2} \kappa}{\sinh \kappa(g+1) \sinh \kappa(g+2)}\left(n_{j, 1} n_{j, 2} n_{j, 3}+n_{j+1,1} n_{j+1,2} n_{j+1,3}\right),
\end{gathered}
$$

where $g, \kappa$ are two free parameters, $n_{j}=n_{j, 1}+n_{j, 2}+n_{j, 3}$ with $n_{j, \alpha}=c_{j, \alpha}^{\dagger} c_{j, \alpha}$ being the number operator for the electron of species $\alpha$ at site $j, \theta(\beta-\alpha)$ is a step function of $(\beta-\alpha)$ and

$$
\eta=-\ln \frac{\sinh \kappa g}{\sinh \kappa(g+1)}, \quad \zeta=\frac{1}{2} \ln \frac{\sinh ^{2} \kappa(g+1)}{\sinh \kappa g \sinh \kappa(g+2)}, \quad \xi=-\ln \frac{\sinh \kappa g}{\sinh \kappa(g+2)}
$$


As is shown in [9], the symmetry algebra underlying (2.2) is the quantum superalgebra $U_{q}[g l(3 \mid 1)]$. The parameter $\kappa$ is related to the deformed parameter $q$ by $q=e^{\kappa}$.

We propose the following nine classes of boundary conditions:

Case (i)

$$
\begin{aligned}
H_{l t}^{\text {boundary }}= & \frac{\sinh \kappa g}{\sinh \frac{\xi_{-}^{I}}{2} \kappa}\left(e^{-\left(\xi_{-}^{I} / 2\right) \kappa} n_{1}-\frac{\sinh \kappa}{\sinh \left(1+\frac{\xi_{-}^{I}}{2}\right) \kappa}\left(n_{1,1} n_{1,2}+n_{1,2} n_{1,3}+n_{1,1} n_{1,3}\right)\right. \\
& \left.+\frac{2 \sinh ^{2} \kappa \cosh \left(1+\frac{\xi_{-}^{I}}{2}\right) \kappa}{\sinh \left(1+\frac{\xi_{-}^{I}}{2}\right) \kappa \sinh \left(2+\frac{\xi_{-}^{I}}{2}\right) \kappa} n_{1,1} n_{1,2} n_{1,3}\right) \\
H_{r t}^{\text {boundary }}= & \frac{\sinh \kappa g}{\sinh \frac{\xi_{+}^{I}}{2} \kappa}\left(e^{\left(\xi_{+}^{I} / 2\right) \kappa} n_{L}-\frac{\sinh \kappa}{\sinh \left(1+\frac{\xi_{+}^{I}}{2}\right) \kappa}\left(n_{L, 1} n_{L, 2}+n_{L, 2} n_{L, 3}+n_{L, 1} n_{L, 3}\right)\right. \\
& \left.+\frac{2 \sinh ^{2} \kappa \cosh \left(1+\frac{\xi_{+}^{I}}{2}\right) \kappa}{\sinh \left(1+\frac{\xi_{+}^{I}}{2}\right) \kappa \sinh \left(2+\frac{\xi_{+}^{I}}{2}\right) \kappa} n_{L, 1} n_{L, 2} n_{L, 3}\right)
\end{aligned}
$$

CASE (ii)

$$
\begin{aligned}
& H_{l t}^{\text {boundary }}=\frac{\sinh \kappa g}{\sinh \frac{\xi_{-}^{I I}}{2} \kappa}\left(e^{-\left(\xi_{-}^{I I} / 2\right) \kappa}\left(n_{1,2}+n_{1,3}\right)-\frac{\sinh \kappa}{\sinh \left(1+\frac{\xi_{-}^{I I}}{2}\right) \kappa} n_{1,2} n_{1,3}\right) \\
& H_{r t}^{\text {boundary }}=\frac{\sinh \kappa g}{\sinh \frac{\xi_{+}^{I I}}{2} \kappa}\left(e^{\left(\xi_{+}^{I I} / 2\right) \kappa}\left(n_{L, 2}+n_{L, 3}\right)-\frac{\sinh \kappa}{\sinh \left(1+\frac{\xi_{+}^{I I}}{2}\right) \kappa} n_{L, 2} n_{L, 3}\right)
\end{aligned}
$$

Case (iii)

$$
\begin{aligned}
& H_{l t}^{\text {boundary }}=\frac{\sinh \kappa g}{\sinh \frac{\xi_{-}^{I I}}{2} \kappa} e^{-\left(\xi_{-}^{I \prime \prime} / 2\right) \kappa} n_{1,3}, \\
& H_{r t}^{\text {boundary }}=\frac{\sinh \kappa g}{\sinh \frac{\xi_{+}^{\prime \prime}}{2} \kappa} e^{\left(\xi_{+}^{\prime \prime \prime} / 2\right) \kappa} n_{L, 3}
\end{aligned}
$$

CASE (iv)

$$
\begin{aligned}
H_{l t}^{\text {boundary }}= & \frac{\sinh \kappa g}{\sinh \frac{\xi_{-}^{I}}{2} \kappa}\left(e^{-\left(\xi_{-}^{I} / 2\right) \kappa} n_{1}-\frac{\sinh \kappa}{\sinh \left(1+\frac{\xi_{-}^{I}}{2}\right) \kappa}\left(n_{1,1} n_{1,2}+n_{1,2} n_{1,3}+n_{1,1} n_{1,3}\right)\right. \\
& \left.+\frac{2 \sinh ^{2} \kappa \cosh \left(1+\frac{\xi_{-}^{I}}{2}\right) \kappa}{\sinh \left(1+\frac{\xi_{-}^{I}}{2}\right) \kappa \sinh \left(2+\frac{\xi_{-}^{I}}{2}\right) \kappa} n_{1,1} n_{1,2} n_{1,3}\right) \\
H_{r t}^{\text {boundary }=} & \frac{\sinh \kappa g}{\sinh \frac{\xi_{+}^{I I}}{2} \kappa}\left(e^{\left(\xi_{+}^{I I} / 2\right) \kappa}\left(n_{L, 2}+n_{L, 3}\right)-\frac{\sinh \kappa}{\sinh \left(1+\frac{\xi_{+}^{I I}}{2}\right) \kappa} n_{L, 2} n_{L, 3}\right)
\end{aligned}
$$


Case (v)

$$
\begin{aligned}
H_{l t}^{\text {boundary }}= & \frac{\sinh \kappa g}{\sinh \frac{\xi_{I}^{I I}}{2} \kappa}\left(e^{-\left(\xi_{-}^{I I} / 2\right) \kappa}\left(n_{1,2}+n_{1,3}\right)-\frac{\sinh \kappa}{\sinh \left(1+\frac{\xi_{-}^{I I}}{2}\right) \kappa} n_{1,2} n_{1,3}\right) \\
H_{r t}^{\text {boundary }}= & \frac{\sinh \kappa g}{\sinh \frac{\xi_{+}^{I}}{2} \kappa}\left(e^{\left(\xi_{+}^{I} / 2\right) \kappa} n_{L}-\frac{\sinh \kappa}{\sinh \left(1+\frac{\xi_{+}^{I}}{2}\right) \kappa}\left(n_{L, 1} n_{L, 2}+n_{L, 2} n_{L, 3}+n_{L, 1} n_{L, 3}\right)\right. \\
& \left.+\frac{2 \sinh ^{2} \kappa \cosh \left(1+\frac{\xi_{+}^{I}}{2}\right) \kappa}{\sinh \left(1+\frac{\xi_{+}^{I}}{2}\right) \kappa \sinh \left(2+\frac{\xi_{+}^{I}}{2}\right) \kappa} n_{L, 1} n_{L, 2} n_{L, 3}\right)
\end{aligned}
$$

CASE (vi)

$$
\begin{aligned}
& \text { (2.9) } H_{l t}^{\text {boundary }}=\frac{\sinh \kappa g}{\sinh \frac{\xi_{-}^{I}}{2} \kappa}\left(e^{-\left(\xi_{-}^{I} / 2\right) \kappa} n_{1}-\frac{\sinh \kappa}{\sinh \left(1+\frac{\xi^{I}}{2}\right) \kappa}\left(n_{1,1} n_{1,2}+n_{1,2} n_{1,3}+n_{1,1} n_{1,3}\right)\right. \\
& \left.+\frac{2 \sinh ^{2} \kappa \cosh \left(1+\frac{\xi_{-}^{I}}{2}\right) \kappa}{\sinh \left(1+\frac{\xi_{I}^{I}}{2}\right) \kappa \sinh \left(2+\frac{\xi_{-}^{I}}{2}\right) \kappa} n_{1,1} n_{1,2} n_{1,3}\right) \text {, } \\
& H_{r t}^{\text {boundary }}=\frac{\sinh \kappa g}{\sinh \frac{\xi_{+}^{I I}}{2} \kappa} e^{\left(\xi_{+}^{I \prime \prime} / 2\right) \kappa} n_{L, 3} \text {; }
\end{aligned}
$$

CASE (vii)

$$
\begin{aligned}
H^{\text {boundary } y_{t i}=} & \frac{\sinh \kappa g}{\sinh \frac{\xi_{-}^{I I I}}{2} \kappa} e^{-\left(\xi_{-}^{I I I} / 2\right) \kappa} n_{1,3} \\
H_{r t}^{\text {boundary }}= & \frac{\sinh \kappa g}{\sinh \frac{\xi_{+}^{I}}{2} \kappa}\left(e^{\left(\xi_{+}^{I} / 2\right) \kappa} n_{L}-\frac{\sinh \kappa}{\sinh \left(1+\frac{\xi_{+}^{I}}{2}\right) \kappa}\left(n_{L, 1} n_{L, 2}+n_{L, 2} n_{L, 3}+n_{L, 1} n_{L, 3}\right)\right. \\
& \left.+\frac{2 \sinh ^{2} \kappa \cosh \left(1+\frac{\xi_{+}^{I}}{2}\right) \kappa}{\sinh \left(1+\frac{\xi_{+}^{I}}{2}\right) \kappa \sinh \left(2+\frac{\xi_{+}^{I}}{2}\right) \kappa} n_{L, 1} n_{L, 2} n_{L, 3}\right)
\end{aligned}
$$

CASE (viii)
(2.11) $H_{l t}^{\text {boundary }}=\frac{\sinh \kappa g}{\sinh \frac{\xi_{I}^{\prime \prime}}{2} \kappa}\left(e^{-\left(\xi_{-}^{I I} / 2\right) \kappa}\left(n_{1,2}+n_{1,3}\right)-\frac{\sinh \kappa}{\sinh \left(1+\frac{\xi_{I}^{\prime \prime}}{2}\right) \kappa} n_{1,2} n_{1,3}\right)$,

$$
H_{r t}^{\text {boundary }}=\frac{\sinh \kappa g}{\sinh \frac{\xi_{+}^{\prime \prime \prime}}{2} \kappa} e^{\left(\xi_{+}^{\prime \prime \prime} / 2\right) \kappa} n_{L, 3}
$$


CASE (ix)

$$
\begin{aligned}
& H_{l t}^{\text {boundary }}=\frac{\sinh \kappa g}{\sinh \frac{\xi_{-}^{I I I}}{2} \kappa} e^{-\left(\xi_{-}^{I I} / 2\right) \kappa} n_{1,3} \\
& H_{r t}^{\text {boundary }}=\frac{\sinh \kappa g}{\sinh \frac{\xi_{ \pm}^{\prime I}}{2} \kappa}\left(e^{\left(\xi_{+}^{I I} / 2\right) \kappa}\left(n_{L, 2}+n_{L, 3}\right)-\frac{\sinh \kappa}{\sinh \left(1+\frac{\xi_{+}^{I I}}{2}\right) \kappa} n_{L, 2} n_{L, 3}\right)
\end{aligned}
$$

where $\xi_{ \pm}^{a}(a=I, I I, I I I)$ are some parameters describing the boundary effects. As will be shown in next section, all nine classes of boundary conditions are integrable.

\section{BOUNDARY K-MATRICES AND QUANTUM INTEGRABILITY}

Quantum integrability of the boundary conditions (2.4-2.12) can be established by means of the (graded) boundary QISM recently formulated in [4]. We first search for boundary K-matrices which satisfy the graded reflection equations:

$$
\begin{aligned}
& R_{12}\left(u_{1}-u_{2}\right) \stackrel{1}{K}-\left(u_{1}\right) R_{21}\left(u_{1}+u_{2}\right) \stackrel{2}{K}-\left(u_{2}\right) \\
& =\stackrel{2}{K}-\left(u_{2}\right) R_{12}\left(u_{1}+u_{2}\right) \stackrel{1}{K}-\left(u_{1}\right) R_{21}\left(u_{1}-u_{2}\right), \\
& R_{21}^{s t_{1} i s t_{2}}\left(-u_{1}+u_{2}\right) \stackrel{1}{K_{+}^{s t_{1}}}\left(u_{1}\right) R_{12}\left(-u_{1}-u_{2}+4\right) K_{+}^{2}{ }^{i s t_{2}}\left(u_{2}\right) \\
& =\stackrel{2}{K_{+}^{i s t_{2}}}\left(u_{2}\right) R_{21}\left(-u_{1}-u_{2}+4\right) \stackrel{1}{K_{+}^{s t_{1}}}\left(u_{1}\right) R_{12}^{s t_{1} i s t_{2}}\left(-u_{1}+u_{2}\right) \text {, }
\end{aligned}
$$

where $R(u) \in E n d(V \otimes V)$, with $V$ being an 8-dimensional representation of $U_{q}[g l(3 \mid 1)]$, is the R-matrix of the two-parameter eight-state supersymmetric fermion model [9], and $R_{21}(u)=P_{12} R_{12}(u) P_{12}$ with $P$ being the graded permutation operator; the supertransposition $s t_{\mu}(\mu=1,2)$ is only carried out in the $\mu$-th factor superspace of $V \otimes V$, whereas $i s t_{\mu}$ denotes the inverse operation of $s t_{\mu}$.

The whole procedure of solving the reflection equations is quite involved. We shall not spell out the details, but state that there are three different diagonal boundary $\mathrm{K}$ matrices, $K_{-}^{I}(u), K_{-}^{I I}(u), K_{-}^{I I I}(u)$, which solve the first reflection equation (3.1):

$$
\begin{aligned}
K_{-}^{I}(u)= & \frac{1}{\sinh \frac{\xi_{-}^{I}}{2} \kappa \sinh \left(1+\frac{\xi_{-}^{I}}{2}\right) \kappa \sinh \left(2+\frac{\xi_{-}^{I}}{2}\right) \kappa} \\
(3.3) \quad & \operatorname{diag}\left(A_{-}^{I}(u), B_{-}^{I}(u), B_{-}^{I}(u), B_{-}^{I}(u), C_{-}^{I}(u), C_{-}^{I}(u), C_{-}^{I}(u), D_{-}^{I}(u)\right), \\
K_{-}^{I I}(u)= & \frac{1}{\sinh \frac{\xi_{-}^{I I}}{2} \kappa \sinh \left(1+\frac{\xi_{-}^{I I}}{2}\right) \kappa} \\
& \operatorname{diag}\left(A_{-}^{I I}(u), A_{-}^{I I}(u), B_{-}^{I I}(u), B_{-}^{I I}(u), B_{-}^{I I}(u), B_{-}^{I I}(u), C_{-}^{I I}(u), C_{-}^{I I}(u)\right), \\
K_{-}^{I I I}(u)= & \frac{1}{\sinh \frac{\xi_{-}^{I I I}}{2} \kappa} \\
& \operatorname{diag}\left(A_{-}^{I I I}(u), A_{-}^{I I I}(u), A_{-}^{I I I}(u), B_{-}^{I I I}(u), A_{-}^{I I I}(u), B_{-}^{I I I}(u), B_{-}^{I I I}(u), B_{-}^{I I I}(u)\right),
\end{aligned}
$$


where

$$
\begin{aligned}
& A_{-}^{I}(u)=-e^{(3 / 2) u \kappa} \sinh \frac{-\xi_{-}^{I}+u}{2} \kappa \sinh \frac{-2-\xi_{-}^{I}+u}{2} \kappa \sinh \frac{-4-\xi_{-}^{I}+u}{2} \kappa, \\
& B_{-}^{I}(u)=e^{(u \kappa / 2)} \sinh \frac{\xi_{-}^{I}+u}{2} \kappa \sinh \frac{-2-\xi_{-}^{I}+u}{2} \kappa \sinh \frac{-4-\xi_{-}^{I}+u}{2} \kappa, \\
& C_{-}^{I}(u)=-e^{-(u \kappa / 2)} \sinh \frac{\xi_{-}^{I}+u}{2} \kappa \sinh \frac{2+\xi_{-}^{I}+u}{2} \kappa \sinh \frac{-4-\xi_{-}^{I}+u}{2} \kappa, \\
& D_{-}^{I}(u)=e^{-(3 / 2) u \kappa} \sinh \frac{\xi_{-}^{I}+u}{2} \kappa \sinh \frac{2+\xi_{-}^{I}+u}{2} \kappa \sinh \frac{4+\xi_{-}^{I}+u}{2} \kappa, \\
& A_{-}^{I I}(u)=e^{u \kappa} \sinh \frac{-\xi_{-}^{I I}+u}{2} \kappa \sinh \frac{-2-\xi_{-}^{I I}+u}{2} \kappa, \\
& B_{-}^{I I}(u)=-\sinh \frac{\xi_{-}^{I I}+u}{2} \kappa \sinh \frac{-2-\xi_{-}^{I I}+u}{2} \kappa, \\
& C_{-}^{I I}(u)=e^{-u \kappa} \sinh \frac{\xi_{-}^{I I}+u}{2} \kappa \sinh \frac{2+\xi_{-}^{I I}+u}{2} \kappa, \\
& A_{-}^{I I I}(u)=-e^{(u / 2) \kappa} \sinh \frac{-\xi_{-}^{I I I}+u}{2} \kappa, \quad B_{-}^{I I I}(u)=e^{-(u / 2) \kappa} \sinh \frac{\xi_{-}^{I I I}+u}{2} \kappa .
\end{aligned}
$$

The corresponding K-matrices, $K_{+}^{I}(u), K_{+}^{I I}(u), K_{+}^{I I I}(u)$, can be obtained from the isomorphism of the two reflection equations. Indeed, given a solution $K_{-}^{a}(u)$ of $(3.1)$, then $K_{+}^{a}(u)$ defined by

$$
K_{+}^{a s t}(u)=M K_{-}^{a}(-u+2), \quad a=I, I I, I I I
$$

are solutions of (3.2). The proof follows from some algebraic computations upon substituting (3.5) into (3.2) and making use of the properties of the R-matrix. Here $M$ is the so-called crossing matrix, which is given in the present case by,

$$
M=\operatorname{diag}\left(1,1, e^{2 \kappa}, e^{4 \kappa}, e^{2 \kappa}, e^{4 \kappa}, e^{6 \kappa}, e^{6 \kappa}\right)
$$

Therefore, one may choose the boundary matrices $K_{+}^{a}(u)$ as

$$
\begin{gathered}
K_{+}^{I}(u)=\operatorname{diag}\left(A_{+}^{I}(u), B_{+}^{I}(u), e^{2 \kappa} B_{+}^{I}(u), e^{4 \kappa} B_{+}^{I}(u), C_{+}^{I}(u), e^{2 \kappa} C_{+}^{I}(u), e^{4 \kappa} C_{+}^{I}(u), D_{+}^{I}(u)\right), \\
K_{+}^{I I}(u)=\operatorname{diag}\left(A_{+}^{I I}(u), A_{+}^{I I}(u), B_{+}^{I I}(u), C_{+}^{I I}(u), B_{+}^{I I}(u), C_{+}^{I I}(u), D_{+}^{I I}(u), D_{+}^{I I}(u)\right), \\
K_{+}^{I I I}(u)=\operatorname{diag}\left(A_{+}^{I I I}(u), A_{+}^{I I I}(u), e^{2 \kappa} A_{+}^{I I I}(u), B_{+}^{I I I}(u), e^{2 \kappa} A_{+}^{I I I}(u)\right. \\
\left.B_{+}^{I I I}(u), e^{2 \kappa} B_{+}^{I I I}(u), e^{2 \kappa} B_{+}^{I I I}(u)\right),
\end{gathered}
$$

where

$$
\begin{aligned}
& A_{+}^{I}(u)=e^{-(3 / 2) u \kappa} \sinh \frac{2 g-\xi_{+}^{I}+u}{2} \kappa \sinh \frac{2 g-2-\xi_{+}^{I}+u}{2} \kappa \sinh \frac{2 g-4-\xi_{+}^{I}+u}{2} \kappa \\
& B_{+}^{I}(u)=e^{-(u+2 / 2) \kappa} \sinh \frac{2 g-\xi_{+}^{I}-u}{2} \kappa \sinh \frac{2 g-2-\xi_{+}^{I}+u}{2} \kappa \sinh \frac{2 g-\xi_{+}^{I}+u}{2} \kappa
\end{aligned}
$$




$$
\begin{aligned}
& C_{+}^{I}(u)=e^{(u-2 / 2) \kappa} \sinh \frac{2 g-\xi_{+}^{I}-u}{2} \kappa \sinh \frac{2 g+2-\xi_{+}^{I}-u}{2} \kappa \sinh \frac{2 g-\xi_{+}^{I}+u}{2} \kappa, \\
& D_{+}^{I}(u)=e^{(3 / 2) u \kappa} \sinh \frac{2 g-\xi_{+}^{I}-u}{2} \kappa \sinh \frac{2 g+2-\xi_{+}^{I}-u}{2} \kappa \sinh \frac{2 g+4-\xi_{+}^{I}-u}{2} \kappa, \\
& A_{+}^{I I}(u)=e^{-u \kappa} \sinh \frac{2 g-\xi_{+}^{I I}+u}{2} \kappa \sinh \frac{2 g-2-\xi_{+}^{I I}+u}{2} \kappa, \\
& B_{+}^{I I}(u)=e^{\kappa} \sinh \frac{2 g-2-\xi_{+}^{I I}+u}{2} \kappa \sinh \frac{2 g+2-\xi_{+}^{I I}-u}{2} \kappa, \\
& C_{+}^{I I}(u)=e^{2 \kappa} \sinh \frac{2 g-\xi_{+}^{I I}+u}{2} \kappa \sinh \frac{2 g+2-\xi_{+}^{I I}-u}{2} \kappa, \\
& D_{+}^{I I}(u)=e^{(u+2) \kappa} \sinh \frac{2 g+2-\xi_{+}^{I I}-u}{2} \kappa \sinh \frac{2 g+4-\xi_{+}^{I I}-u}{2} \kappa, \\
& A_{+}^{I I I}(u)=e^{-u \kappa / 2} \sinh \frac{2 g-\xi_{+}^{I I}+u}{2} \kappa, \\
& B_{+}^{I I I}(u)=e^{(u+2 / 2) \kappa} \sinh \frac{2 g+4-\xi_{+}^{I I I}-u}{2} \kappa,
\end{aligned}
$$

Following Sklyanin's arguments [15], one may show that the quantity $\mathcal{T}_{-}(u)$ given by

$$
\mathcal{T}_{-}(u)=T(u) K_{-}(u) T^{-1}(-u), \quad T(u)=R_{0 L}(u) \cdots R_{01}(u),
$$

satisfies the same relation as $K_{-}(u)$ :

$$
\begin{aligned}
R_{12}\left(u_{1}-u_{2}\right) \stackrel{1}{\mathcal{T}}_{-}\left(u_{1}\right) R_{21}\left(u_{1}\right. & \left.+u_{2}\right) \stackrel{2}{\mathcal{T}}-\left(u_{2}\right) \\
& =\stackrel{2}{\mathcal{T}}_{-}\left(u_{2}\right) R_{12}\left(u_{1}+u_{2}\right) \stackrel{1}{\mathcal{T}}-\left(u_{1}\right) R_{21}\left(u_{1}-u_{2}\right)
\end{aligned}
$$

Thus if one defines the boundary transfer matrix $\tau(u)$ as

$$
\tau(u)=\operatorname{str}\left(K_{+}(u) \mathcal{T}_{-}(u)\right)=\operatorname{str}\left(K_{+}(u) T(u) K_{-}(u) T^{-1}(-u)\right)
$$

then it can be shown [4] that $\left[\tau\left(u_{1}\right), \tau\left(u_{2}\right)\right]=0$. Since $K_{ \pm}(u)$ can be taken as $K_{ \pm}^{I}(u), K_{ \pm}^{I I}(u)$ and $K_{ \pm}^{I I I}(u)$, respectively, we have nine possible choices of the boundary transfer matrices:

$$
\tau^{(a, b)}(u)=\operatorname{str}\left(K_{+}^{a}(u) T(u) K_{-}^{b}(u) T^{-1}(-u)\right), \quad a, b=I, I I, I I I,
$$

which reflects the fact that the boundary conditions on the left end and on the right end of the open lattice chain are independent.

Now it can be shown that Hamiltonians corresponding to all nine boundary conditions are related to the second derivative of the boundary transfer matrix $\tau^{(a, b)}(u$ ) (up to an unimportant additive constant) 


$$
\begin{aligned}
& \text { (3.13) } H=\frac{2 \sinh \kappa g}{\kappa} H^{(a, b)} \text {, } \\
& H^{(a, b)}=\frac{\tau^{(a, b))^{\prime \prime}}(0)}{4(V+2 W)}=\sum_{j=1}^{L-1} H_{j, j+1}^{R}+\frac{1}{2}{\stackrel{1}{K^{b}}}^{\prime}-(0)+\frac{1}{2(V+2 W)}\left[s t r_{0}\left(\stackrel{0}{K^{a}}+(0) G_{L 0}\right)\right. \\
& \left.+2 \operatorname{str} 0\left(\stackrel{0}{K^{a^{\prime}}}+(0) H_{L 0}^{R}\right)+\operatorname{str} 0\left(\stackrel{0}{K^{a}}+(0)\left(H_{L 0}^{R}\right)^{2}\right)\right] \text {, }
\end{aligned}
$$

where

$$
\begin{gathered}
V=s t r_{0} K_{+}^{a^{\prime}}(0), \quad W=s \operatorname{st}_{0}\left(\stackrel{0}{K}^{a}+(0) H_{L 0}^{R}\right), \\
H_{j, j+1}^{R}=P_{j, j+1} R_{j, j+1}^{\prime}(0), \quad G_{j, j+1}=P_{j, j+1} R_{j, j+1}^{\prime \prime}(0) .
\end{gathered}
$$

Here $P_{j, j+1}$ denotes the graded permutation operator acting on the $j$-th and $j+1$-th quantum spaces. (3.14) implies that the boundary two-parameter eight-state supersymmetric models admit an infinite number of conservation currents which are in involution with each other, thus assuring their quantum integrability.

\section{Coordinate Bethe Ansatz Analysis}

Having established the quantum integrability of the boundary models, we now solve them by using the coordinate space Bethe ansatz method. Following $[1,14,16,4]$, we assume that the eigenfunction of the Hamiltonian (2.2) takes the form

$$
\begin{gathered}
|\Psi\rangle=\sum_{\left\{\left(x_{j}, \alpha_{j}\right)\right\}} \Psi_{\alpha_{1}, \ldots, \alpha_{N}}\left(x_{1}, \ldots, x_{N}\right) c_{x_{1} \alpha_{1}}^{\dagger} \ldots c_{x_{N} \alpha_{N}}^{\dagger}|0\rangle, \\
\Psi_{\alpha_{1}, \ldots, \alpha_{N}}\left(x_{1}, \ldots, x_{N}\right)=\sum_{P} \varepsilon_{P} A_{\alpha_{Q 1}, \ldots, \alpha_{Q N}}\left(k_{P Q 1}, \ldots, k_{P Q N}\right) \exp \left(i \sum_{j=1}^{N} k_{P_{j}} x_{j}\right),
\end{gathered}
$$

where the summation is taken over all permutations and negations of $k_{1}, \ldots, k_{N}$, and $Q$ is the permutation of the $N$ particles such that $1 \leqslant x_{Q 1} \leqslant \cdots \leqslant x_{Q N} \leqslant L$. The symbol $\varepsilon_{P}$ is a sign factor \pm 1 and changes its sign under each 'mutation'. On substituting the wavefunction into the eigenvalue equation $H|\Psi\rangle=E|\Psi\rangle$, one gets

$$
\begin{aligned}
A_{\ldots, \alpha_{j}, \alpha_{i}, \ldots}\left(\ldots, k_{j}, k_{i}, \ldots\right) & =S_{i j}\left(k_{i}, k_{j}\right) A_{\ldots, \alpha_{i}, \alpha_{j}, \ldots}\left(\ldots, k_{i}, k_{j}, \ldots\right), \\
A_{\alpha_{i}, \ldots}\left(-k_{j}, \ldots\right) & =s^{L}\left(k_{j} ; p_{1 \alpha_{i}}\right) A_{\alpha_{i}, \ldots}\left(k_{j}, \ldots\right), \\
A_{\ldots, \alpha_{i}}\left(\ldots,-k_{j}\right) & =s^{R}\left(k_{j} ; p_{L \alpha_{i}}\right) A_{\ldots, \alpha_{i}}\left(\ldots, k_{j}\right),
\end{aligned}
$$

where $S_{i j}\left(k_{i}, k_{j}\right)$ are the two-particle scattering matrices,

$$
\begin{aligned}
S_{i j}\left(k_{i}, k_{j}\right)_{a a}^{a a} & =1, \quad a=1,2,3, \\
S_{i j}\left(k_{i}, k_{j}\right)_{a b}^{a b} & =\frac{\sin \left(\lambda_{i}-\lambda_{j}\right)}{\sin \left(\lambda_{i}-\lambda_{j}-i \kappa\right)}, \quad a \neq b, a, b=1,2,3, \\
S_{i j}\left(k_{i}, k_{j}\right)_{b a}^{a b} & =e^{i \operatorname{sign}(a-b)\left(\lambda_{i}-\lambda_{j}\right)} \frac{\sin i \kappa}{\sin \left(\lambda_{i}-\lambda_{j}-i \kappa\right)}, \quad a, b=1,2,3,
\end{aligned}
$$


where $\lambda_{j}$ are suitable particle rapidities related to the quasi-momenta $k_{j}$ of the electrons by

$$
k(\lambda)=2 \arctan (\operatorname{coth} c \tan \lambda)
$$

where the parameter $c$ is defined by

$$
c=\frac{1}{4}\left\{\ln \left[\frac{\sinh \frac{1}{2}(\eta+\kappa)}{\sinh \frac{1}{2}(\eta-\kappa)}\right]-\kappa\right\} .
$$

$s^{L}\left(k_{j} ; p_{1 \alpha_{i}}\right)$ and $s^{R}\left(k_{j} ; p_{L \alpha_{i}}\right)$ are the boundary scattering matrices,

$$
\begin{aligned}
s^{L}\left(k_{j} ; p_{1 \alpha_{i}}\right) & =\frac{1-p_{1 \alpha_{i}} e^{i k_{j}}}{1-p_{1 \alpha_{i}} e^{-i k_{j}}} \\
s^{R}\left(k_{j} ; p_{L \alpha_{i}}\right) & =\frac{1-p_{L \alpha_{i}} e^{-i k_{j}}}{1-p_{L \alpha_{i}} e^{i k_{j}}} e^{2 i k_{j}(L+1)}
\end{aligned}
$$

with $p_{1 \alpha_{i}}$ and $p_{L \alpha_{i}}$ being given by the following formulae, corresponding to the nine cases respectively,

CASE (i)

$$
\begin{aligned}
& p_{1,1}=p_{1,2}=p_{1,3} \equiv p_{1}=-e^{-\kappa g}+e^{-\left(\xi_{-}^{I} / 2\right) \kappa} \frac{\sinh \kappa g}{\sinh \frac{\xi_{-}^{I}}{2} \kappa} \\
& p_{L, 1}=p_{L, 2}=p_{L, 3} \equiv p_{L}=-e^{-\kappa g}+e^{\left(\xi_{+}^{I} / 2\right) \kappa} \frac{\sinh \kappa g}{\sinh \frac{\xi_{+}^{I}}{2} \kappa}
\end{aligned}
$$

CAse (ii)

$$
\begin{aligned}
& p_{1,1}=-e^{-\kappa g}, \quad p_{1,2}=p_{1,3}=-e^{-\kappa g}+e^{-\left(\xi_{-}^{I \prime} / 2\right) \kappa} \frac{\sinh \kappa g}{\sinh \frac{\xi_{-}^{I I}}{2} \kappa} \\
& p_{L, 1}=-e^{-\kappa g}, \quad p_{L, 2}=p_{L, 3}=-e^{-\kappa g}+e^{\left(\xi_{+}^{I I} / 2\right) \kappa} \frac{\sinh \kappa g}{\sinh \frac{\xi_{+}^{I I}}{2} \kappa}
\end{aligned}
$$

CASE (iii)

$$
\begin{aligned}
& p_{1,1}=p_{1,2}=-e^{-\kappa g}, \quad p_{1,3}=-e^{-\kappa g}+e^{-\left(\xi_{-}^{I I} / 2\right) \kappa} \frac{\sinh \kappa g}{\sinh \frac{\xi_{-}^{I I I}}{2} \kappa} \\
& p_{L, 1}=p_{L, 2}=-e^{-\kappa g}, \quad p_{L, 3}=-e^{-\kappa g}+e^{\left(\xi_{+}^{I I I} / 2\right) \kappa} \frac{\sinh \kappa g}{\sinh \frac{\xi_{+}^{I I}}{2} \kappa}
\end{aligned}
$$

Case (iv)

$$
\begin{aligned}
& p_{1,1}=p_{1,2}=p_{1,3} \equiv p_{1}=-e^{-\kappa g}+e^{-\left(\xi_{-}^{I} / 2\right) \kappa} \frac{\sinh \kappa g}{\sinh \frac{\xi_{-}^{I}}{2} \kappa} \\
& p_{L, 1}=-e^{-\kappa g}, \quad p_{L, 2}=p_{L, 3}=-e^{-\kappa g}+e^{\left(\xi_{+}^{I J} / 2\right) \kappa} \frac{\sinh \kappa g}{\sinh \frac{\xi_{+}^{I I}}{2} \kappa}
\end{aligned}
$$


Case (v)

$$
\begin{aligned}
& p_{1,1}=-e^{-\kappa g}, \quad p_{1,2}=p_{1,3}=-e^{-\kappa g}+e^{-\left(\xi_{-}^{I I} / 2\right) \kappa} \frac{\sinh \kappa g}{\sinh \frac{\xi_{-}^{I I}}{2} \kappa} \\
& p_{L, 1}=p_{L, 2}=p_{L, 3} \equiv p_{L}=-e^{-\kappa g}+e^{\left(\xi_{+}^{I} / 2\right) \kappa} \frac{\sinh \kappa g}{\sinh \frac{\xi_{+}^{I}}{2} \kappa}
\end{aligned}
$$

Case (vi)

$$
\begin{aligned}
& p_{1,1}=p_{1,2}=p_{1,3} \equiv p_{1}=-e^{-\kappa g}+e^{-\left(\xi_{-}^{I} / 2\right) \kappa} \frac{\sinh \kappa g}{\sinh \frac{\xi_{-}^{I}}{2} \kappa} \\
& p_{L, 1}=p_{L, 2}=-e^{-\kappa g}, \quad p_{L, 3}=-e^{-\kappa g}+e^{\left(\xi_{+}^{I I} / 2\right) \kappa} \frac{\sinh \kappa g}{\sinh \frac{\xi_{+}^{I I}}{2} \kappa} ;
\end{aligned}
$$

Case (vii)

$$
\begin{aligned}
& p_{1,1}=p_{1,2}=-e^{-\kappa g}, \quad p_{1,3}=-e^{-\kappa g}+e^{-\left(\xi_{-}^{I \prime \prime} / 2\right) \kappa} \frac{\sinh \kappa g}{\sinh \frac{\xi_{-}^{I I}}{2} \kappa} \\
& p_{L, 1}=p_{L, 2}=p_{L, 3} \equiv p_{L}=-e^{-\kappa g}+e^{\left(\xi_{+}^{I} / 2\right) \kappa} \frac{\sinh \kappa g}{\sinh \frac{\xi_{+}^{I}}{2} \kappa}
\end{aligned}
$$

CAse (viii)

$$
\begin{aligned}
& p_{1,1}=-e^{-\kappa g}, \quad p_{1,2}=p_{1,3}=-e^{-\kappa g}+e^{-\left(\xi_{-}^{I I} / 2\right) \kappa} \frac{\sinh \kappa g}{\sinh \frac{\xi_{-}^{I I}}{2} \kappa} \\
& p_{L, 1}=p_{L, 2}=-e^{-\kappa g}, \quad p_{L, 3}=-e^{-\kappa g}+e^{\left(\xi_{+}^{I I} / 2\right) \kappa} \frac{\sinh \kappa g}{\sinh \frac{\xi_{+}^{I I}}{2} \kappa}
\end{aligned}
$$

CASE (ix)

$$
\begin{aligned}
& p_{1,1}=p_{1,2}=-e^{-\kappa g}, \quad p_{1,3}=-e^{-\kappa g}+e^{-\left(\xi_{-}^{I I} / 2\right) \kappa} \frac{\sinh \kappa g}{\sinh \frac{\xi_{-}^{I I}}{2} \kappa}, \\
& p_{L, 1}=-e^{-\kappa g}, \quad p_{L, 2}=p_{L, 3}=-e^{-\kappa g}+e^{\left(\xi_{+}^{I I} / 2\right) \kappa} \frac{\sinh \kappa g}{\sinh \frac{\xi_{+}^{I I}}{2} \kappa} .
\end{aligned}
$$

As is seen above, the two-particle S-matrix (4.3) is nothing but the R-matrix of the $U_{q}[g l(3)]$-invariant Heisenberg magnetic chain and thus satisfies the quantum YangBaxter equation (QYBE),

$$
S_{i j}\left(k_{i}, k_{j}\right) S_{i l}\left(k_{i}, k_{l}\right) S_{j l}\left(k_{j}, k_{l}\right)=S_{j l}\left(k_{j}, k_{l}\right) S_{i l}\left(k_{i}, k_{l}\right) S_{i j}\left(k_{i}, k_{j}\right) .
$$

It can be checked that the boundary scattering matrices $s^{L}$ and $s^{R}$ obey the reflection equations:

$$
\begin{aligned}
S_{j i}\left(-k_{j},-k_{i}\right) s^{L}\left(k_{j} ; p_{1 a_{j}}\right) & S_{i j}\left(-k_{i}, k_{j}\right) s^{L}\left(k_{i} ; p_{1 \alpha_{i}}\right) \\
= & s^{L}\left(k_{i} ; p_{1 \alpha_{i}}\right) S_{j i}\left(-k_{j}, k_{i}\right) s^{L}\left(k_{j} ; p_{1 \alpha_{i}}\right) S_{i j}\left(k_{i}, k_{j}\right) \\
S_{j i}\left(-k_{j},-k_{i}\right) s^{R}\left(k_{j} ; p_{L \alpha_{j}}\right) & S_{i j}\left(k_{i},-k_{j}\right) s^{R}\left(k_{i} ; p_{L \alpha_{i}}\right) \\
= & s^{R}\left(k_{i} ; p_{L \alpha_{i}}\right) S_{j i}\left(k_{j},-k_{i}\right) s^{R}\left(\left(k_{j} ; p_{L \alpha_{i}}\right) ; p_{\alpha_{i}}\right) S_{j i}\left(k_{j}, k_{i}\right) .
\end{aligned}
$$


This is seen as follows. One introduces the notation

$$
s(k ; p)=\frac{1-p e^{-i k}}{1-p e^{i k}} .
$$

Then the boundary scattering matrices $s^{L}\left(k_{j} ; p_{1 \alpha_{i}}\right), s^{R}\left(k_{j} ; p_{L \alpha_{i}}\right)$ can be written as, corresponding to the nine cases respectively,

CAse (i)

$$
\begin{aligned}
& s^{L}\left(k_{j} ; p_{1 \alpha_{i}}\right)=s\left(-k_{j} ; p_{1}\right) I, \\
& s^{R}\left(k_{j} ; p_{L \alpha_{i}}\right)=e^{i k_{j} 2(L+1)} s\left(k_{j} ; p_{L}\right) I
\end{aligned}
$$

CASE (ii)

$$
\begin{aligned}
& \text { (4.20) } s^{L}\left(k_{j} ; p_{1 \alpha_{i}}\right)=s\left(-k_{j} ; p_{1,1}\right)\left(\begin{array}{ccc}
1 & 0 & 0 \\
0 & e^{2 i \lambda_{j}} \frac{\sin \left(\zeta_{-}+\lambda_{j}\right)}{\sin \left(\zeta_{-}-\lambda_{j}\right)} & 0 \\
0 & 0 & e^{2 i \lambda_{j}} \frac{\sin \left(\zeta_{-}+\lambda_{j}\right)}{\sin \left(\zeta_{-}-\lambda_{j}\right)}
\end{array}\right) \text {, } \\
& s^{R}\left(k_{j} ; p_{L a_{i}}\right)=e^{i k_{j} 2(L+1)} s\left(k_{j} ; p_{L, 1}\right)\left(\begin{array}{ccc}
1 & 0 & 0 \\
0 & e^{2 i \lambda_{j}} \frac{\sin \left(\zeta_{+}-\lambda_{j}\right)}{\sin \left(\zeta_{+}+\lambda_{j}\right)} & 0 \\
0 & 0 & e^{2 i \lambda_{j}} \frac{\sin \left(\zeta_{+}-\lambda_{j}\right)}{\sin \left(\zeta_{+}+\lambda_{j}\right)}
\end{array}\right)
\end{aligned}
$$

CASE (iii)

$$
\begin{aligned}
& s^{L}\left(k_{j} ; p_{1 \alpha_{i}}\right)=s\left(-k_{j} ; p_{1,1}\right)\left(\begin{array}{ccc}
1 & 0 & 0 \\
0 & 1 & 0 \\
0 & 0 & e^{2 i \lambda_{j}} \frac{\sin \left(\zeta_{-}^{\prime}+\lambda_{j}\right)}{\sin \left(\zeta_{-}^{\prime}-\lambda_{j}\right)}
\end{array}\right) \\
& s^{R}\left(k_{j} ; p_{L \alpha_{i}}\right)=e^{i k_{j} 2(L+1)} s\left(k_{j} ; p_{L, 1}\right) \\
& \left(\begin{array}{ccc}
1 & 0 & 0 \\
0 & 1 & 0 \\
0 & 0 & e^{2 i \lambda_{j}} \frac{\sin \left(\zeta_{+}^{\prime}-\lambda_{j}\right)}{\sin \left(\zeta_{+}^{\prime}+\lambda_{j}\right)}
\end{array}\right)
\end{aligned}
$$

CASE (iv)

$$
\begin{aligned}
& s^{L}\left(k_{j} ; p_{1 \alpha_{1}}\right)=s\left(-k_{j} ; p_{1}\right) I \\
& s^{R}\left(k_{j} ; p_{L \alpha_{i}}\right)=e^{i k_{j} 2(L+1)} s\left(k_{j} ; p_{L, 1}\right)\left(\begin{array}{ccc}
1 & 0 & 0 \\
0 & e^{2 i \lambda_{j}} \frac{\sin \left(\zeta_{+}-\lambda_{j}\right)}{\sin \left(\zeta_{+}+\lambda_{j}\right)} & 0 \\
0 & 0 & e^{2 i \lambda_{j}} \frac{\sin \left(\zeta_{+}-\lambda_{j}\right)}{\sin \left(\zeta_{+}+\lambda_{j}\right)}
\end{array}\right)
\end{aligned}
$$


CASE (v)

$$
\begin{aligned}
s^{L}\left(k_{j} ; p_{1 \alpha_{i}}\right) & =s\left(-k_{j} ; p_{1,1}\right) \\
s^{R}\left(k_{j} ; p_{L \alpha_{i}}\right) & =e^{i k_{j} 2(L+1)} s\left(k_{j} ; p_{L}\right) I
\end{aligned}
$$

CASE (vi)

$$
\begin{aligned}
& s^{L}\left(k_{j} ; p_{1 \alpha_{i}}\right)=s\left(-k_{j} ; p_{1}\right) I \\
& s^{R}\left(k_{j} ; p_{L \alpha_{i}}\right)=e^{i k_{j} 2(L+1)} s\left(k_{j} ; p_{L, 1}\right)\left(\begin{array}{ccc}
1 & 0 & 0 \\
0 & 1 & 0 \\
0 & 0 & e^{2 i \lambda_{j}} \frac{\sin \left(\zeta_{+}^{\prime}-\lambda_{j}\right)}{\sin \left(\zeta_{+}^{\prime}+\lambda_{j}\right)}
\end{array}\right)
\end{aligned}
$$

CASE (vii)

$$
\begin{aligned}
& s^{L}\left(k_{j} ; p_{1 \alpha_{i}}\right)=s\left(-k_{j} ; p_{1,1}\right)\left(\begin{array}{ccc}
1 & 0 & 0 \\
0 & 1 & 0 \\
0 & 0 & e^{2 i \lambda_{j}} \frac{\sin \left(\zeta_{-}^{\prime}+\lambda_{j}\right)}{\sin \left(\zeta_{-}^{\prime}-\lambda_{j}\right)}
\end{array}\right), \\
& s^{R}\left(k_{j} ; p_{L \alpha_{i}}\right)=e^{i k_{j} 2(L+1)} s\left(k_{j} ; p_{L}\right) I
\end{aligned}
$$

CASE (viii)

$$
\begin{aligned}
& \text { (4.26) } \quad s^{L}\left(k_{j} ; p_{1 \alpha_{i}}\right)=s\left(-k_{j} ; p_{1,1}\right)\left(\begin{array}{ccc}
1 & 0 & 0 \\
0 & e^{2 i \lambda_{j}} \frac{\sin \left(\zeta_{-}+\lambda_{j}\right)}{\sin \left(\zeta_{-}-\lambda_{j}\right)} & 0 \\
0 & 0 & e^{2 i \lambda_{j}} \frac{\sin \left(\zeta_{-}+\lambda_{j}\right)}{\sin \left(\zeta_{-}-\lambda_{j}\right)}
\end{array}\right) \text {, } \\
& s^{R}\left(k_{j} ; p_{L \alpha_{i}}\right)=e^{i k_{j} 2(L+1)} s\left(k_{j} ; p_{L, 1}\right)\left(\begin{array}{ccc}
1 & 0 & 0 \\
0 & 1 & 0 \\
0 & 0 & e^{2 i \lambda_{j}} \frac{\sin \left(\zeta_{+}^{\prime}-\lambda_{j}\right)}{\sin \left(\zeta_{+}^{\prime}+\lambda_{j}\right)}
\end{array}\right)
\end{aligned}
$$


CASE (ix)

$$
\begin{aligned}
& s^{L}\left(k_{j} ; p_{1 \alpha_{i}}\right)=s\left(-k_{j} ; p_{1,1}\right)\left(\begin{array}{ccc}
1 & 0 & 0 \\
0 & 1 & 0 \\
0 & 0 & e^{2 i \lambda_{j}} \frac{\sin \left(\zeta_{-}^{\prime}+\lambda_{j}\right)}{\sin \left(\zeta_{-}^{\prime}-\lambda_{j}\right)}
\end{array}\right), \\
& s^{R}\left(k_{j} ; p_{L \alpha_{i}}\right)=e^{i k_{j} 2(L+1)} s\left(k_{j} ; p_{L, 1}\right)\left(\begin{array}{ccc}
1 & 0 & 0 \\
0 & e^{2 i \lambda_{j}} \frac{\sin \left(\zeta_{+}-\lambda_{j}\right)}{\sin \left(\zeta_{+}+\lambda_{j}\right)} & 0 \\
0 & 0 & e^{2 i \lambda_{j}} \frac{\sin \left(\zeta_{+}-\lambda_{j}\right)}{\sin \left(\zeta_{+}+\lambda_{j}\right)}
\end{array}\right) \text {. }
\end{aligned}
$$

Here $I$ stands for the $3 \times 3$ identity matrix and $p_{1,1}, p_{L, 1}$ are as given in $(4.8) ; \zeta_{ \pm}, \zeta_{ \pm}^{\prime}$ are parameters defined by

$$
\zeta_{ \pm}=\frac{g-\xi_{ \pm}^{I I}}{2 i} \kappa, \quad \zeta_{ \pm}^{\prime}=\frac{g-\xi_{ \pm}^{I I I}}{2 i} \kappa
$$

We immediately see that (4.19) are the trivial solutions of the reflection equations (4.17), whereas (4.20) and (4.21) are the diagonal solutions $[15,13,7]$.

The diagonalisation of the Hamiltonian (2.2) reduces to solving the following matrix eigenvalue equation

$$
T_{j} t=t, \quad j=1, \ldots, N,
$$

where $t$ denotes an eigenvector on the space of the spin variables and $T_{j}$ takes the form

$$
T_{j}=S_{j}^{-}\left(k_{j}\right) s^{L}\left(-k_{j} ; p_{1 \sigma_{j}}\right) R_{j}^{-}\left(k_{j}\right) R_{j}^{+}\left(k_{j}\right) s^{R}\left(k_{j} ; p_{L \sigma_{j}}\right) S_{j}^{+}\left(k_{j}\right)
$$

with

$$
\begin{aligned}
S_{j}^{+}\left(k_{j}\right) & =S_{j, N}\left(k_{j}, k_{N}\right) \ldots S_{j, j+1}\left(k_{j}, k_{j+1}\right), \\
S_{j}^{-}\left(k_{j}\right) & =S_{j, j-1}\left(k_{j}, k_{j-1}\right) \ldots S_{j, 1}\left(k_{j}, k_{1}\right), \\
R_{j}^{-}\left(k_{j}\right) & =S_{1, j}\left(k_{1},-k_{j}\right) \ldots S_{j-1, j}\left(k_{j-1},-k_{j}\right), \\
R_{j}^{+}\left(k_{j}\right) & =S_{j+1, j}\left(k_{j+1},-k_{j}\right) \ldots S_{N, j}\left(k_{N},-k_{j}\right) .
\end{aligned}
$$

This problem can be solved using the algebraic Bethe ansatz method. The Bethe ansatz equations for all the nine cases are

$$
\begin{aligned}
& e^{i k_{j} 2(L+1)} F\left(k_{j} ; p_{1,1}, p_{L, 1}\right)=\prod_{\sigma=1}^{M_{1}} \frac{\sin \left(\lambda_{j}-\Lambda_{\sigma}^{(1)}+i \kappa / 2\right)}{\sin \left(\lambda_{j}-\Lambda_{\sigma}^{(1)}-i \kappa / 2\right)} \frac{\sin \left(\lambda_{j}+\Lambda_{\sigma}^{(1)}+i \kappa / 2\right)}{\sin \left(\lambda_{j}+\Lambda_{\sigma}^{(1)}-i \kappa / 2\right)}, \\
& \prod_{j=1}^{N} \frac{\sin \left(\Lambda_{\sigma}^{(1)}-\lambda_{j}+i \kappa / 2\right)}{\sin \left(\Lambda_{\sigma}^{(1)}-\lambda_{j}-i \kappa / 2\right)} \frac{\sin \left(\Lambda_{\sigma}^{(1)}+\lambda_{j}+i \kappa / 2\right)}{\sin \left(\Lambda_{\sigma}^{(1)}+\lambda_{j}-i \kappa / 2\right)}
\end{aligned}
$$




$$
\begin{array}{r}
=G\left(\Lambda_{\sigma}^{(1)} ; \zeta_{-}, \zeta_{+}\right) \prod_{\substack{\rho=1 \\
\rho \neq \sigma}}^{M_{1}} \frac{\sin \left(\Lambda_{\sigma}^{(1)}-\Lambda_{\rho}^{(1)}+i \kappa\right)}{\sin \left(\Lambda_{\sigma}^{(1)}-\Lambda_{\rho}^{(1)}-i \kappa\right)} \frac{\sin \left(\Lambda_{\sigma}^{(1)}-\Lambda_{\rho}^{(1)}+i \kappa\right)}{\sin \left(\Lambda_{\sigma}^{(1)}-\Lambda_{\rho}^{(1)}-i \kappa\right)} \\
\prod_{\rho=1}^{M_{2}} \frac{\sin \left(\Lambda_{\sigma}^{(1)}-\Lambda_{\rho}^{(2)}-i \kappa / 2\right)}{\sin \left(\Lambda_{\sigma}^{(1)}-\Lambda_{\rho}^{(2)}+i \kappa / 2\right)} \frac{\sin \left(\Lambda_{\sigma}^{(1)}+\Lambda_{\rho}^{(2)}-i \kappa / 2\right)}{\sin \left(\Lambda_{\sigma}^{(1)}+\Lambda_{\rho}^{(2)}+i \kappa / 2\right)}, \sigma=1, \ldots, M_{1}, \\
\prod_{\rho=1}^{M_{1}} \frac{\sin \left(\Lambda_{\gamma}^{(2)}-\Lambda_{\rho}^{(1)}+i \kappa / 2\right)}{\sin \left(\Lambda_{\gamma}^{(2)}-\Lambda_{\rho}^{(1)}-i \kappa / 2\right)} \frac{\sin \left(\Lambda_{\gamma}^{(2)}+\Lambda_{\rho}^{(1)}+i \kappa / 2\right)}{\sin \left(\Lambda_{-}^{(2)} \gamma+L_{\rho}^{(1)}-i \kappa / 2\right)} \\
=K\left(\Lambda^{(2)} ; \zeta_{-}^{\prime}, \zeta_{+}^{\prime}\right) \prod_{\substack{\rho=1 \\
\rho \neq \gamma}}^{M_{2}} \frac{\sin \left(\Lambda_{\gamma}^{(2)}-\Lambda_{\rho}^{(2)}+i \kappa\right)}{\sin \left(\Lambda_{\gamma}^{(2)}-\Lambda_{\rho}^{(2)}-i \kappa\right)} \frac{\sin \left(\Lambda_{\gamma}^{(2)}+\Lambda_{\rho}^{(2)}+i \kappa\right)}{\sin \left(\Lambda_{\gamma}^{(2)}+\Lambda_{\rho}^{(2)}-i \kappa\right)}, \\
\gamma=1, \ldots, M_{2},
\end{array}
$$

where

(4.33) $F\left(k_{j} ; p_{1,1}, p_{L, 1}\right)=s\left(k_{j} ; p_{1,1}\right) s\left(k_{j} ; p_{L, 1}\right), \quad$ (for all cases),

$$
G\left(\Lambda_{\sigma}^{(1)} ; \zeta_{-}, \zeta_{+}\right)= \begin{cases}\frac{1}{\sin \left(\zeta_{-}+\Lambda_{\sigma}^{(1)}+\frac{i \kappa}{2}\right)} \frac{\sin \left(\zeta_{+}+\Lambda_{\sigma}^{(1)}+\frac{i \kappa}{2}\right)}{\sin \left(\zeta_{-}-\Lambda_{\sigma}^{(1)}+\frac{i \kappa}{2}\right)} \text { case (i) } \\ \sin \left(\zeta_{+}-\Lambda_{\sigma}^{(1)}+\frac{i \kappa}{2}\right) & \text { case (ii) } \\ \frac{\sin \left(\zeta_{+}+\Lambda_{\sigma}^{(1)}+\frac{i \kappa}{2}\right)}{\sin \left(\zeta_{+}-\Lambda_{\sigma}^{(1)}+\frac{i \kappa}{2}\right)} e^{2 i \Lambda_{\sigma}^{(1)}} & \text { case (iii) } \\ \frac{\sin \left(\zeta_{-}+\Lambda_{\sigma}^{(1)}+\frac{i \kappa}{2}\right)}{\sin \left(\zeta_{-}-\Lambda_{\sigma}^{(1)}+\frac{i \kappa}{2}\right)} e^{-2 i \Lambda_{\sigma}^{(1)}} & \text { case (iv) } \\ 1 & \text { case (v) } \\ 1 & \text { case (vi) } \\ \frac{\sin \left(\zeta_{-}+\Lambda_{\sigma}^{(1)}+\frac{i \kappa}{2}\right)}{\sin \left(\zeta_{-}-\Lambda_{\sigma}^{(1)}+\frac{i \kappa}{2}\right)} e^{-2 i \Lambda_{\sigma}^{(1)}} & \text { case (vii) } \\ \frac{\sin \left(\zeta_{+}+\Lambda_{\sigma}^{(1)}+\frac{i \kappa}{2}\right)}{\sin \left(\zeta_{+}-\Lambda_{\sigma}^{(1)}+\frac{i \kappa}{2}\right)} e^{2 i \Lambda_{\sigma}^{(1)}} & \text { case (viii) }\end{cases}
$$




$$
K\left(\Lambda^{(2)} ; \zeta_{-}^{\prime}, \zeta_{+}^{\prime}\right)= \begin{cases}1 & \text { case (i) } \\ 1 & \text { case (ii) } \\ \frac{\sin \left(\zeta_{-}^{\prime}+\Lambda_{\gamma}^{(2)}+i \kappa\right)}{\sin \left(\zeta_{-}^{\prime}-\Lambda_{\gamma}^{(2)}+i \kappa\right)} \frac{\sin \left(\zeta_{+}^{\prime}+\Lambda_{\gamma}^{(2)}+i \kappa\right)}{\sin \left(\zeta_{+}^{\prime}-\Lambda_{\gamma}^{(2)}+i \kappa\right)} & \text { case (iii) } \\ 1 & \text { case (iv) } \\ 1 & \text { case (v) } \\ \frac{\sin \left(\zeta_{+}^{\prime}+\Lambda_{\gamma}^{(2)}+i \kappa\right)}{\sin \left(\zeta_{+}^{\prime}-\Lambda_{\gamma}^{(2)}+i \kappa\right)} e^{2 i \Lambda_{\gamma}^{(2)}} & \text { case (vi) } \\ \frac{\sin \left(\zeta_{-}^{\prime}+\Lambda_{\gamma}^{(2)}+i \kappa\right)}{\sin \left(\zeta_{-}^{\prime}-\Lambda_{\gamma}^{(2)}+i \kappa\right)} e^{-2 i \Lambda_{\gamma}^{(2)}} & \text { case (vii) } \\ \frac{\sin \left(\zeta_{+}^{\prime}+\Lambda_{\gamma}^{(2)}+i \kappa\right)}{\sin \left(\zeta_{+}^{\prime}-\Lambda_{\gamma}^{(2)}+i \kappa\right)} e^{2 i \Lambda_{\gamma}^{(2)}} & \text { case (viii) } \\ \frac{\sin \left(\zeta_{-}^{\prime}+\Lambda_{\gamma}^{(2)}+i \kappa\right)}{\sin \left(\zeta_{-}^{\prime}-\Lambda_{\gamma}^{(2)}+i \kappa\right)} e^{-2 i \Lambda_{\gamma}^{(2)}} & \text { case (ix) }\end{cases}
$$

The energy eigenvalue $E$ of the model is given by $E=-2 \sum_{j=1}^{N} \cos k_{j}$ (modulo an unimportant additive constant coming from the chemical potential term).

\section{REFERENCES}

[1] H. Asakawa and M. Suzuki, 'Finite-size corrections in the $X X X$ model and the Hubbard model with boundary fields', J. Phys. A 29 (1996), 225-145.

[2] R.Z. Bariev, 'Integrable model of interacting $X Y$ chains', J. Phys. A 24 (1991), L919-L923.

[3] R.Z. Bariev, A. Klümper and J. Zittartz, 'A new integrable two-parameter model of strongly correlated electrons in one-dimension', Europhys. Lett. 32 (1995), 85-90.

[4] A.J. Bracken, X.-Y. Ge, Y.-Z. Zhang and H.-Q. Zhou, 'Integrable open-boundary conditions for the $q$-deformed super symmetric $U$ model of strongly correlated electrons', Nuclear Phys. B 516 (1998), 588-602.

[5] A.J. Bracken, X.-Y. Ge, Y.-Z. Zhang and H.-Q. Zhou, 'An open-boundary integrable model of three coupled $X Y$ spin chains', Nuclear Phys. B 516 (1998), 603-622.

[6] A.J. Bracken, M.D. Gould, J.R. Links and Y.-Z. Zhang, 'New supersymmetric and exactly solvable model of correlated electrons', Phys. Rev. Lett. 74 (1995), 2768-2771.

[7] H.J. de Vega and A. González-Ruiz, 'Boundary $K$-matrices for the six vertex and the $n(2 n-1) A_{n-1}$ vertex models', J. Phys. A 26 (1993), L519-L524.

[8] F.H.L. Essler and V.E. Korepin, Exactly solvable models of strongly correlated electrons (World Scientific, Singapore, 1994).

[9] X.-Y. Ge, M.D. Gould, Y.-Z. Zhang and H.-Q. Zhou, 'A new two-parameter integrable model of strongly correlated fermions with quantum superalgegra symmetry', J. Phys. A 31 (1998), 5233-5239. 
[10] A. González-Ruiz, 'Integrable open-boundary conditions for the supersymmetric $T-J$ model', Nuclear Phys. B 424 (1994), 468-486.

[11] M.D. Gould, K.E. Hibberd, J.R. Links and Y.-Z. Zhang, 'Integrable electron model with correlated hopping and quantum supersymmetry', Phys. Lett. A 212 (1996), 156-160.

[12] M.D. Gould, Y.-Z. Zhang and H.-Q. Zhou, 'Eight-state supersymmetric $U$ model of strongly correlated fermions', Phys. Rev. B 57 (1998), 9498-9501.

[13] L. Mezincescu and R.I. Nepomechie, 'Integrable open spin chains with non-symmetric R-matrices', J. Phys. A 24 (1991), L17-L23.

[14] M. Shiroishi, M. Wadati, 'Bethe ansatz equation for the Hubbard model with boundary fields', J. Phys. Soc. Japan 66 (1997), 1-4.

[15] E.K. Sklyanin, 'Boundary conditions for integrable quantum sytems', J. Phys. A 21 (1988), 2375-2389.

[16] Y.-Z. Zhang and H.-Q. Zhou, 'Quantum integrability and exact solution of the supersymmetric $U$ model with boundary terms', Phys. Rev. B 58 (1998), 51-53.

[17] Y.-Z. Zhang and H.-Q. Zhou, 'New integrable boundary conditions for the $q$-deformed supersymmetric $U$ model and Bethe ansatz equations', Phys. Lett. A 244 (1998), 427-431.

[18] H.-Q. Zhou, 'Quantum integrability for the one-dimensional Hubbard open chain', Phys. Rev. B 54 (1996), 41-43.

[19] H.-Q. Zhou, 'Integrable open-boundary conditions for the one-dimensional Bariev chain', Phys. Rev. B 53 (1996), 5098-5100.

Department of Mathematics

The University of Queensland

Queensland 4072

Australia

e-mail: ajb@maths.uq.edu.au xg@maths.uq.edu.au yzz@maths.uq.edu.au hqz@maths.uq.edu.au 